\title{
Sexual Function the First Year Postpartum: A Mini-Commentary
}

\author{
Rachel Pope ${ }^{1}$ \\ ${ }^{1}$ University Hospitals Cleveland Medical Center
}

September 25, 2021

Sexual Function the First Year Postpartum: A Mini-Commentary

Rachel Pope MD, MPH

Urology Insitute, Division of Female Sexual Health

University Hospitals, Cleveland Medical Center

11100 Euclid Avenue, Cleveland, OH 44106

Rachel.pope@uhhospitals.org

Running Title: Postpartum Sexual Function

In the first year postpartum, women tend to be burdened by physical exhaustion due to interrupted and lack of sleep, breastfeeding and the potential challenges therein, and chemically-induced anxiety and depression. Literature in the field of behavioral health has long-since described the high prevalence of postpartum mental health disturbances of $20 \%$ of all women, and the impact of mental health on quality of life documented world-wide (O'Hara MW et al. Perinatal mental illness: definition, description and aetiology. Best Pract Res Clin Obstet Gynaecol. 2014 Jan;28(1):3-12). Sexual dysfunction, however, is a lesser-known challenge and one that is notably under-reported and addressed in the medical literature, likely due to other dominating discomforts and an overall stigmatization of female sexual health. Furthermore, scheduled visits with medical providers rarely extend beyond six weeks postpartum and most women have not resumed sexual activity at this time.

Sexual function is an important aspect of quality of life. There is straight-forward treatment for dysfunction and dyspareunia that may be caused by hypo-oestrogenized tissues and pelvic floor injuries. Cattani et al. highlight the experiences of women around the world through a comprehensive systematic review. It is not surprising then, that obstetric anal sphincter injuries (OASIS), episiotomies, and instrumental vaginal birth are all associated with sexual dysfunction and/or dyspareunia. While anal sphincter injuries affect approximately $6 \%$ of women (Jha S et al. Risk factors for recurrent obstetric anal sphincter injury (rOASI): a systematic review and meta-analysis. Int Urogynecol J. 2016 Jun;27(6):849-57.) and episiotomies continue to be on the decline, the paper by Cattani et al does show less dyspareunia among women who deliver by cesarean compared to spontaneous vaginal birth. One might assume this is related to the pelvic floor injuries. However, mode of delivery is not associated with overall sexual dysfunction. Strikingly, OASIS was associated with an odds ratio of 3 (1.28-7.03) for sexual dysfunction and 1.92 (1.47-2.52) for dyspareunia. While these injuries are not easily preventable, these data inform and strengthen the need for specialized clinics, follow-up care and increased attention to individuals who have sustained them (Madsen A, Hickman L, and Propst K. Recognition and Management of Pelvic Floor Disorders in Pregnancy and the Postpartum Period, Obstetrics and Gynecology Clinics of North America. 2021. 48; (3):571-584). For example, if an individual with a third or fourth degree laceration is identified as having increased risk for pain and dysfunction sexually, she should be counseled on this as to empower her to seek care should the concern 
arise. Lubricants, vaginal estrogens and DHEA can all be appropriately prescribed even if breastfeeding and could greatly improve her experience (Donders GGG, et al. Pharmacotherapy for the treatment of vaginal atrophy. Expert Opin Pharmacother. 2019 May;20(7):821-835.). Furthermore, this information underscores the need for women who have sustained a higher order laceration to present for pelvic floor physical therapy and rehabilitate as a preventive and therapeutic measure. Specialized clinics can help patients navigate this.

Vaginal dryness from lactation is extremely common. This review only identified one study on vaginal dryness and sexual concerns. This does not indicate that vaginal dryness is not a problem, but rather that more research is indicated. Another unexplored variable is urinary incontinence. A large proportion of women experience urinary incontinence in the first year postpartum. It would be worth exploring the connection between UI and sexual dysfunction.

Therefore, while this review represents progress in understanding the mechanism of sexual dysfunction and dyspareunia the first year postpartum, there is still more to be learned in the form of empiric evidence, especially regarding vaginal dryness and urinary incontinence. 